Artículo. Soledad Aravena, Riva Quiroga. "Desarrollo léxico tardío en textos explicativos de estudiantes secundarios: análisis de adjetivos".

\title{
DESARROLLO LÉXICO TARDÍO EN TEXTOS EXPLICATIVOS DE ESTUDIANTES SECUNDARIOS: ANÁLISIS DE ADJETIVOS
}

\author{
LATER LEXICAL DEVELOPMENT IN EXPLANATIONS \\ BY HIGH SCHOOL STUDENTS: ANALYSIS OF ADJECTIVES
}

\author{
Soledad Aravena \\ Pontificia Universidad Católica de Chile \\ maravena@uc.cl \\ Riva Quiroga \\ Pontificia Universidad Católica de Chile \\ riva.quiroga@uc.cl
}

\begin{abstract}
RESUMEN
La investigación se propuso explorar evolutivamente los adjetivos, una clase gramatical que ofrece una perspectiva óptima para estudiar el desarrollo léxico y lingüístico en edades escolares. Se recolectaron 120 textos explicativos escritos por estudiantes de tres niveles escolares (séptimo básico y primero y tercero medio) y dos grupos socioeconómicos. En ellos fueron contabilizados todos los adjetivos y analizados también algunos de sus rasgos morfológicos, sintácticos y semánticos. Para el procesamiento de los datos se aplicó un diseño de ANOVA factorial mixto de dos factores intergrupales (edad/nivel escolar y grupo socioeconómico). Los resultados indican que la edad y su interacción con el grupo socioeconómico inciden significativamente en el uso total de adjetivos y en el de adjetivos derivados, prenominales, modificadores y relacionales. Se concluye que el léxico puede experimentar un gran incremento en estas etapas de acuerdo con variables contextuales y de dominio del género.
\end{abstract}

Palabras clave: desarrollo léxico - adjetivos - escritura explicativa

\begin{abstract}
The research aims to explore adjectives, a grammatical class that offers an optimal perspective to study lexical and linguistic development at school ages. 120 explanatory texts written by students from three high school levels and two socioeconomic groups were collected. In them, all the adjectives were counted and some of their morphological, syntactic and semantic features were also analyzed. For data analysis, a mixed factorial ANOVA design with two intergroup factors (age / school level and SES group) was applied. The results show that age / school level and its interaction with the SES group significantly influence the total use of adjectives and the use of derivative, prenominal, modifying and relational adjectives. We concluded that lexicon can greatly increase at these stages according to contextual variables and discourse genre command.
\end{abstract}

Keywords: lexical development - adjectives - explanatory writing

Recibido: 30 de mayo de 2020

Aceptado: 22 de junio de 2020 


\section{INTRODUCCIÓN}

El aprendizaje del léxico constituye un factor crítico en la adquisición de la lengua materna, por su conexión evidente con el desarrollo cognitivo y social de los niños, así como también con el desarrollo de las estructuras gramaticales (Dockrell y Messer 35; Ravid, Semantic Development 792). En efecto, la incorporación de nuevas palabras modifica los dominios conceptuales de los niños, lo que expande sus estructuras de memoria y su capacidad de aprender. Dichos dominios conceptuales se enriquecen con la incorporación de nuevos términos, pero también lo hacen producto de la progresiva sofisticación y delimitación que van adoptando los significados de los términos ya incorporados. En cuanto al desarrollo gramatical, sabemos que el uso de las primeras palabras impulsa la comprensión y expansión de sus estructuras morfológicas y posteriormente también de las estructuras sintácticas (Serrat et al. 445). Estos logros, a su vez, fomentan nuevos usos léxicos, dando así lugar a un proceso de mutuo enriquecimiento en la interfaz léxico-gramatical (Llauradó y Tolchinsky 629). El desarrollo avanzado del vocabulario interactúa así con la complejidad sintáctica y la densidad informativa de los textos, de modo tal que, mientras mayor es la ocurrencia de las palabras de contenido, mayor es la complejidad de las estructuras sintácticas que las contienen (Ravid, Derivational Morphology 54).

Muchos estudios han llevado a pensar, en ocasiones, que el desarrollo léxico es un proceso simple y que no demanda gran esfuerzo. En efecto, los niños pequeños pueden aprender la impresionante tasa de diez palabras por día (Clark 75) y en condiciones experimentales se ha comprobado que usualmente les basta una única exposición a una nueva palabra para incorporarla (Ameel et al. 262). Estas fases iniciales de la adquisición léxica son destinadas principalmente a los sustantivos comunes, concretos y vinculados con la experiencia, o al menos imaginables (Hansen 207). Posteriormente se incorporan los verbos y más tarde los adjetivos (Bloom 6). Así, los niños pueden comprender y usar todas las clases de palabras léxicas con relativa prontitud: hacia los 5-6 años, al término de la etapa del desarrollo léxico temprano, conocen unas 10.000 palabras, aproximadamente (Dockrell y Messer 43). Sin embargo, diversos estudios recientes sobre los desarrollos tardíos del léxico han demostrado que alcanzar un dominio proficiente y flexible de los significados les ocupa toda la infancia y algunos años de la adolescencia (Ravid, Derivational Morphology 80; Tolchinsky 234). Al respecto, todavía no existe demasiada claridad acerca del modo en que los niños mayores logran incorporar el vasto vocabulario que aun les queda por aprender, unas 50.000 palabras en total al término de la escolaridad (Cutillas, L'ús dels adjectius 27; Lively et al. 269). 
Artículo. Soledad Aravena, Riva Quiroga. "Desarrollo léxico tardío en textos explicativos de estudiantes secundarios: análisis de adjetivos".

En este contexto, el presente trabajo se propone analizar los adjetivos, una categoría léxica habitualmente poco estudiada en los trabajos evolutivos. Los adjetivos son expresiones más generales que verbos y sustantivos, por lo que conforman un grupo de recursos menos indispensables y, por lo tanto, más opcionales y retóricos. Por otro lado, debido a su relación de dependencia, son dúctiles sintácticamente (ya que pueden adoptar distintas funciones y posiciones en el grupo nominal, como veremos a continuación), y muestran comportamientos muy variados y complejos desde el punto de vista léxico-conceptual (Demonte 134; McNally y Kennedy 1). Esta complejidad sintáctica y semántica permite a los adjetivos enriquecer el significado de las expresiones linguiísticas y los convierte en recursos muy pertinentes para observar los usos léxicos durante la escolaridad, la etapa de los llamados desarrollos tardíos del lenguaje (Cutillas y Tolchinsky 59; Nippold 3).

\section{Conceptos operativos para un estudio evolutivo de los adjetivos}

Siguiendo la definición que proporciona la gramática descriptiva (Demonte 133; RAE 235), se entenderán los adjetivos como una clase de palabra con determinadas características morfológicas, sintácticas y semánticas, que modifica al sustantivo, denotando sus propiedades o cualidades y aportando significados diversos acerca del modo en que se mencionan y relacionan las entidades (Demonte 133; Bosque 3). Para el presente estudio, el adjetivo será considerado en un sentido restrictivo, es decir, como elemento léxico de clase abierta, excluyendo cuantificadores, numerales, determinantes y otros adjetivos de escaso contenido léxico como algunos comparativos. Además de los adjetivos léxicos propios (como "luminoso"), se incluyen en este análisis los sustantivos adjetivados (como en la expresión "programa espía") y los participios con función adjetiva (como en "texto editado") (Millán 207; RAE 525).

Las dimensiones del adjetivo que se considerarán son su estructura morfológica, su posición en la frase nominal, su función sintáctica y algunos de sus rasgos semánticos, particularmente, si se usan con un sentido calificativo o relacional. En cuanto a la morfología, es una dimensión que ha demostrado ser pertinente desde el punto de vista evolutivo, dado que el contacto de los niños con palabras derivadas se ha relacionado con un mayor volumen de palabras y de conocimientos lingüísticos, particularmente semánticos y sintácticos (Anglin et al. 36; Tyler y Nagy 650). Se espera por lo tanto que los adjetivos derivados sean más frecuentes en los textos de los estudiantes mayores. La posición del adjetivo en el grupo nominal, en tanto, puede ser anterior al sustantivo (adjetivos prenominales); o posterior al mismo (adjetivos posnominales). Esta última es la posición no marcada y más frecuente en castellano, asumida por 
el adjetivo típicamente especificativo (Bosque y Picallo 367; Cuadrado 85). Por ello, se presume que será la posición adjetival más frecuente en todo el corpus y la más utilizada por los estudiantes menores. Los adjetivos prenominales en cambio no especifican ni restringen la extensión del sustantivo, sino que más bien se usan para destacar o evaluar un rasgo de su significado (como en "inquietante espera" o "alegre reencuentro") (Cuadrado 85). Por ello, estos adjetivos tienen un carácter aun más optativo que los posnominales, que podrían considerarse más necesarios para una descripción que sea suficientemente informativa (como en "persona enferma" o "última oficina").

Para el análisis de la función, los adjetivos pueden ser clasificados como atributos predicativos, en cuyo caso modifican al sustantivo indirectamente mediante un verbo copulativo (como en "es difícil") o no copulativo (como en "parece vacío") o, pueden ser modificadores, en caso de que determinen al sustantivo directamente (como en "odiosa actitud" y "oficina regional") (Demonte 136). Tal como ha sido divulgado en algunos estudios sobre desarrollo léxico (Nelson 15; Ninio 256), la función predicativa del adjetivo es más temprana que la modificadora, puesto que esta última requiere de una integración lógico-semántica de dos etapas más compleja para los niños pequeños. Además, los adjetivos modificadores se enmarcan en construcciones sintácticas que los hacen también más opcionales o retóricos, mientras que los predicativos resultan más obligatorios. Sería esperable, por lo tanto, una mayor presencia de adjetivos modificadores enmarcados en construcciones sintácticas elaboradas entre los estudiantes de los niveles escolares superiores (Ravid, Derivational Morphology 78). Esto se debe a que los sintagmas que contienen a estos tipos de adjetivos tienen, por un lado, gran capacidad de empaquetar información y concentrar densidad informativa y, por el otro, son construcciones sintácticas expandibles recursivamente. Ambas - la densidad informativa y la complejidad sintáctica, en particular la mayor complejidad de la frase nominal- han sido relacionadas con la producción típica de estudiantes mayores y con los usos propios de la escritura y del lenguaje académico (Biber y Gray 167-168).

Por último, en la dimensión semántica del análisis, se distinguirán los adjetivos calificativos de los relacionales. Los primeros indican cualidades constitutivas de las cosas o entidades y que se manifiestan a través de una propiedad única como el tamaño, el color o la forma (Demonte 138-139; RAE 238). Por ejemplo, en una expresión como "gesto tierno" la propiedad que se informa es la ternura del gesto. Los adjetivos relacionales (también llamados categorizadores o descriptivos), en tanto, adscriben no ya una propiedad, sino que a un conjunto de propiedades que modifican al objeto o entidad, poniéndolo en relación con otras entidades, 
Artículo. Soledad Aravena, Riva Quiroga. "Desarrollo léxico tardío en textos explicativos de estudiantes secundarios: análisis de adjetivos".

como en los ejemplos "evento histórico", "artículo constitucional" o "texto escolar". En estos ejemplos, los adjetivos no expresan una sola propiedad, sino que se refieren a un conjunto de propiedades, las que permiten definir los sustantivos historia, constitución y escuela (Bosque 4; Demonte 138-139; RAE 253). Aunque los adjetivos relacionales no constituyen una clase del todo homogénea (Bosque y Picallo 351), en esta investigación se consideran de manera conjunta, cautelando que todos satisfagan tres rasgos fundamentales que los distinguen de los adjetivos calificativos: no pueden ser medibles graduables (*texto muy escolar); no pueden formar parte de relaciones binarias de polaridad (es decir no tienen antónimos), y (generalmente) no pueden ser usados como atributos predicativos (*el artículo es constitucional). Sin embargo, el contexto puede hacer que un adjetivo relacional (ella estudia arte dramático) se utilice como adjetivo calificativo (su despedida fue muy dramática) en cuyo caso puede cumplir con todas las condiciones de los adjetivos calificativos. (Demonte 138-139)

\section{METODOLOGÍA}

El presente es un estudio cuantitativo, descriptivo y cuasi-experimental, enmarcado en el campo de los desarrollos tardíos del lenguaje.

\section{Instrumentos}

Se diseñó una tarea semiespontánea de escritura, elicitada mediante un material audiovisual sobre el tema "Internet y las redes sociales" (tema que había sido el más votado en una encuesta realizada con anterioridad), con un breve contexto retórico e instrucciones para escribir un texto explicativo. El video y la tarea de escritura fueron evaluados por cinco investigadores en escritura, a quienes se les pidió revisarlos en relación con aspectos tales como pertinencia e interés del tema, claridad de instrucciones, suficiencia de la situación retórica, entre otros. En general, los expertos consultados consideraron el material adecuado, pertinente y formulado correctamente, pero sus sugerencias condujeron a una formulación más completa del contexto (situación retórica) y a una redacción más clara de las instrucciones. La tarea fue probada luego en una aplicación piloto $y$, tras leves modificaciones, se logró su versión definitiva, que se presenta a continuación: 


\section{$\underline{\text { Recursos de la Red }}$}

Como viste en el video, Internet ofrece muchos sitios y recursos para conocer nuevas cosas y aprender sobre lo que nos interesa: nos informamos sobre actualidad en la prensa y en las redes sociales, utilizamos buscadores de información y enciclopedias, aprendemos nuevos juegos, creamos y desarrollamos perfiles personales o grupales, compartimos información, música, películas y programas, entre otras posibilidades.

Elige uno de esos sitios o recursos de Internet que te guste y conozcas bien (no importa si no aparece en el video). Una vez elegido, te pedimos escribir un artículo en que lo presentes y lo expliques (por ejemplo, qué es, para qué sirve, cómo se usa, etc.), para personas que no lo conocen y quieren aprender a usarlo.

Tu texto podría aparecer en una revista digital o Blog donde niños y jóvenes aprenden acerca de los usos de Internet. Es importante informar y explicar de la manera más completa posible. Puedes hacer un borrador si lo deseas y utilizar todas las hojas que necesites.

\section{Selección de la muestra}

Originalmente, la muestra incluyó a 480 estudiantes de séptimo básico (12-13 años), primero medio (14-15 años) y tercero medio (16-17 años) pertenecientes a dos establecimientos mixtos de la ciudad de Santiago representativos de los grupos socioeconómicos alto (GS alto) y medio-bajo (GS medio-bajo), según información proporcionada por el Ministerio de Educación. Los rangos etarios fueron seleccionados porque se consideran relevantes para los fenómenos que interesa observar en el estudio y porque pueden informar acerca de conductas de entrada y (próxima) salida de la Enseñanza Media en el sistema escolar chileno. Se mostró el video a los estudiantes y luego se les solicitó escribir dos textos -uno explicativo y otro narrativo (que no será considerado en este estudio)-. Los textos resultantes en esta primera etapa fueron revisados con rúbricas debidamente validadas que permitieron asignarles puntajes de desempeño global. Con este puntaje, se excluyeron de la muestra todos los sujetos con rendimiento sobre y bajo las 1,96 unidades de Desviación Estándar, medida utilizada habitualmente para la detección de los casos atípicos. Hechas las exclusiones, se seleccionaron de manera aleatoria 120 participantes 20 estudiantes de cada nivel y establecimiento (10 hombres y 10 mujeres)-, cuyos 120 textos explicativos conformaron el corpus definitivo del presente estudio. Se decidió trabajar con 120 participantes tras un análisis de potencia estadística calculado en $G^{*}$ Power (Faul et al. 181), que estableció un $N$ muestral mínimo de 108 sujetos. Esta cifra garantizaba óptimas posibilidades de detectar significaciones estadísticas asociadas a un tamaño de efecto cercano al típicamente considerado como mediano $(0,25)$. 
Artículo. Soledad Aravena, Riva Quiroga. "Desarrollo léxico tardío en textos explicativos de estudiantes secundarios: análisis de adjetivos".

\section{Procedimientos de transcripción y segmentación de textos}

Con el fin de asegurar consenso y consistencia en el tratamiento del corpus, se siguieron las orientaciones que la bibliografía especializada sugiere para este tipo de procedimientos (Neuendorf 134; Stemler 6). Así, se elaboró un protocolo de transcripción y un manual de segmentación y etiquetado, y parte del corpus fue sometido a distintas rondas de calibración con el fin de asegurar la consistencia de los investigadores en la aplicación de los criterios definidos, tal como se describe a continuación. Los 120 textos fueron transcritos de forma literal utilizando un procesador de textos y luego convertidos a formato CHAT (*.cha) con el fin de poder realizar los análisis en el programa CLAN (MacWhinney 9), habitual en estudios del área. Preparar los archivos para el análisis requirió hacer algunas modificaciones, como unir locuciones y nombres propios compuestos, con el fin de no alterar el número total de palabras, decisión que también ha sido tomada en otros estudios sobre léxico y para evitar que el análisis morfológico realizado a través de CLAN interpretara erróneamente algunas categorías gramaticales.

\section{Etiquetado de variables y procesamiento de datos}

Si bien se ejecutó el programa MOR de CLAN (que genera un análisis morfológico de cada palabra) para contabilizar diversos fenómenos, en el presente estudio los adjetivos fueron etiquetados manualmente, debido a que era necesario distinguir como adjetivos ciertas palabras y expresiones con función adjetiva que no eran reconocidas como tales por el programa, por ejemplo, las locuciones y algunos participios. Este etiquetado manual consistió en marcar cada uno de los adjetivos (y participios que funcionan como adjetivos) resultando en las siguientes medidas: proporción de adjetivos por texto sobre el total de palabras, proporción de adjetivos simples y derivados (se marcaron las locuciones pero por su escasa frecuencia no serán incluidas en este reporte), proporción de adjetivos en función predicativa y modificadora, proporción de adjetivos prenominales y posnominales y proporción de adjetivos calificativos y relacionales. Para el análisis de los datos se aplicó un diseño de ANOVA factorial mixto con dos factores intergrupo: edad/nivel escolar (en el entendido de que este factor se asocia con distintos grados de experiencia letrada y lingüística en general) y grupo socioeconómico (en el entendido de que este factor se relaciona en Chile con el grado de alfabetización familiar y el tipo de establecimiento y comuna). Las comparaciones post-hoc de todas las pruebas de varianza se realizaron mediante la prueba de Tukey. 


\section{RESULTADOS}

\section{Total de adjetivos}

Tabla 1. Frecuencia absoluta y relativa de adjetivos. Media grupal y desviación estándar (DE).

\begin{tabular}{|c|c|c|c|c|c|c|c|c|c|c|}
\hline \multicolumn{2}{|c|}{$\begin{array}{l}\text { GS y } \\
\text { nivel } \\
\text { escolar }\end{array}$} & $\begin{array}{l}\text { Total de } \\
\text { palabras }\end{array}$ & Mín. & Má & $\begin{array}{c}\text { Total de } \\
\text { adjetivos }\end{array}$ & Mín. & Máx. & $\begin{array}{c}\text { Proporción } \\
\text { de } \\
\text { adjetivos* }\end{array}$ & Mín. & Máx. \\
\hline \multirow{3}{*}{$\mathrm{M}$} & Sép. & $136(61,7)$ & 65 & 257 & $5,0(3,60)$ & 0 & 14 & 0,0348 & 0 & 0,0930 \\
\hline & Pri. & $145(57,9)$ & 56 & 269 & $4,9(2,17)$ & 1 & 9 & 0,0358 & 0,0123 & 0,0714 \\
\hline & Ter. & $201(83,8)$ & 91 & 407 & $7,8(4,43)$ & 1 & 21 & 0,0402 & 0,0062 & 0,0827 \\
\hline \multirow{3}{*}{ A } & Sép. & $177(55,1)$ & 81 & 282 & $5,3(2,41)$ & 1 & 11 & 0,0299 & 0,0084 & 0,0476 \\
\hline & Pri. & $245(71,9)$ & 181 & 400 & $11,5(4,59)$ & 2 & 21 & 0,0485 & 0,0101 & 0,0821 \\
\hline & Ter. & $365(115,9)$ & 172 & 515 & $18,2(5,92)$ & 8 & 27 & 0,0521 & 0,0231 & 0,0952 \\
\hline
\end{tabular}

*Proporción de adjetivos sobre el total de palabras del texto

Los resultados de esta investigación revelan que los estudiantes mayores utilizan un número mayor de adjetivos en sus textos, tal como se puede apreciar en la Tabla 1. La frecuencia absoluta de adjetivos aumenta -indudablemente, puesto que también aumenta la extensión de los textos- entre séptimo y tercero medio, de 5 a 7,8 en el colegio de grupo socioeconómico (GS) medio y de 5,3 a 18,2 en el de GS alto. Lo relevante, sin embargo, es observar que el aumento también se produce en las frecuencias relativas de adjetivos, es decir, en su proporción respecto del número total de palabras del texto. Como se constata en la Tabla 1, esta proporción sube levemente de 0,034 a 0,040 en el colegio de GS medio-bajo y lo hace de manera más marcada en el de GS alto, de 0,029 a 0,052. Estas cifras aparentemente exiguas se traducen en que, en este último establecimiento, los estudiantes menores usan una media de 2,9 adjetivos cada 100 palabras, mientras que los mayores alcanzan una media de 5,2 (una cifra alta, si se piensa por ejemplo que este párrafo tiene 190 palabras, hasta aquí).

Las pruebas de varianza revelan que las diferencias por edad/nivel escolar son estadísticamente significativas $(F(1,114)=7,183 ; p=, 0011)$ y las pruebas posthoc indican que las diferencias se presentan entre los niveles de séptimo y tercero, naturalmente $(p=, 0010)$, pero también entre séptimo y primero $(p=, 0267)$. Esto significa que, si bien las cifras de adjetivos aumentan durante toda la adolescencia, el principal cambio evolutivo para el número de adjetivos en este género se produce entre los 12 y los 14 años. Por otro lado, también resultaron significativas las diferencias entre los dos tipos de colegio $(F(1,114)=4,604 ; p=, 0340)$ y la interacción entre ambos factores (edad/nivel escolar y grupo socioeconómico, $F(2,114)=3,486$; $p=, 0339)$. Las comparaciones posthoc muestran que las diferencias importantes se dan entre 
Artículo. Soledad Aravena, Riva Quiroga. "Desarrollo léxico tardío en textos explicativos de estudiantes secundarios: análisis de adjetivos".

séptimo y tercero del GS alto $(p=, 0007)$ y entre séptimo y primero del mismo establecimiento $(p$ $=, 0007)$. Esta interacción reafirma el hecho de que las trayectorias que experimentan los estudiantes entre séptimo y tercero medio difieren marcadamente en los dos colegios y esta diferencia alcanza a ser significativa no solo respecto del factor edad, sino también respecto del factor GS.

Los siguientes ejemplos ilustran bien algunas de estas diferencias entre los menores y los mayores. Han sido seleccionados principalmente porque tienen poca extensión y representan bien los desempeños de sus respectivos grupos en diversos rasgos, algunos de los cuales se comentarán en los siguientes apartados. Los ejemplos 1 y 2 son de séptimo básico y tienen 3 adjetivos cada uno, es decir, una cifra similar a la media de sus respectivos grupos $(3,4$ adjetivos cada 100 palabras en el GS medio-bajo y 2,9\% en el GS alto). El ejemplo 3 es de tercero medio y tiene 16 adjetivos en 218 palabras (un poco más que la media de su grupo, que es de 5,2 adjetivos en 100 palabras), es decir quintuplica la proporción de adjetivos de los textos precedentes.

Ejemplo (1), séptimo básico, GS medio-bajo (65 palabras, 3 adjetivos).

\section{El Facebook}

Es un medio de comunicación masiva, la utilizamos generalmente para chatear con amigos o familiares, subir fotos o videos. También lo puedes usar para jugar, hacer nuevos amigos, mandar mensajes, etc... El Facebook hay que usarlo con mucho cuidado ya que pueden haber demasiadas personas malas, y por sobre todo debemos tener precaución con las cosas que se escriben en este medio.

Ejemplo (2), séptimo básico, GS alto (134 palabras, 3 adjetivos).

Gmail

Si tu quieres meterte a Gmail, métete a internet y busca Gmail, el Gmail es lo más seguro, cuando te crees su sesión escribe toda la verdad, y si no tienes más de 13 años no te dejan creártelo, no es tan difícil de usar si quieres enviarle un mensaje a un amigo, debes preguntarle cómo se llama su usuario, al saber eso le puedes enviar todo lo que quieras a él, no te confíes de tus amigos y no le digas tu contraseña, porque tal vez ya no puedas usarla nunca más. Esto te sirve para comunicarte con otras personas que están en otro lugar. El Gmail es una página donde te puedes conectar con otras personas, puedes enviarles fotos, hablar de lo que tú quieras pero sin usar mal vocabulario. 
Ejemplo (3), tercero medio, GS alto (218 palabras, 16 adjetivos)

Twitter es una red social que nos permite comunicarnos con otras personas, opinando sobre diversos temas o bien comentar otras opiniones. Twitter permite a los usuarios "seguir" marcas, artistas e incluso figuras de relevancia como puede llegar a ser un reconocido comentarista o incluso un político importante. He allí la gracia de esta red, la comunicación y opinión que se puede generar entre 2 o más individuos. Muchos comparan a Twitter con Facebook inclinándose por este último, pero personalmente difiero de aquellas opiniones al creer que la gran diferencia que existe entre estos es que Twitter genera opinión que no se construye a partir de perfiles y fotos, sino a partir de comentarios personales o de información pública. Twitter ofrece además ser seguidor de importantes diarios y noticieros que informan constantemente hechos de relevancia nacional como mundial. Si bien la utilización de esta red es múltiple en cuanto a la cantidad de recursos que tiene, todo se rige principalmente por los comentarios que se crean, estos pueden incluso relatar una anécdota, dirigida a otra persona, o puede ser también un comentario abierto que se publicará en internet, remitiendo a tus seguidores que lo vean y lo comenten. Twitter retoma la importancia de la crítica y la opinión pública, para hacernos partícipes de comentarios interesantes en ciertas ocasiones y polémicos en otras.

Los ejemplos anteriores -más allá de las evidentes diferencias cuantitativas ya comentadas- también permiten observar el efecto que causan los adjetivos en los textos y las funciones que cumplen en los distintos grupos. En los dos primeros textos, los adjetivos (modificadores y atributos) contribuyen, en parte, a la transmisión de una modalidad deóntica, habitual en los menores (Berman et al. 260). Es decir, sirven para dar juicios de valor en expresiones tales como "Gmail es lo más seguro" o "sin usar mal vocabulario" o "puede haber demasiadas personas malas". Otros usos de los adjetivos en estos textos se dan en expresiones o fórmulas que son altamente frecuentes y que, muy probablemente resultan opciones más accesibles para los niños durante la escritura de un género difícil. Es el caso de "medio de comunicación masiva" (adjetivo relacional, como se comentará más adelante) y "nuevos amigos" (adjetivo prenominal, como también se comentará). En el ejemplo 3, en tanto, los adjetivos son muy diversos y cumplen también diversas funciones (ninguna relacionada con la modalidad deóntica, que es más habitual en los niños menores): dar énfasis como en "reconocido comentarista"; categorizar para destacar, como en "relevancia nacional como mundial", categorizar y especificar, lo que demuestra conocimiento del tema, como en "comentario abierto", y simplemente calificar pero con términos precisos y poco frecuentes que imprimen mayor riqueza léxica al texto, como en "red ...múltiple" y "comentarios ... polémicos". No hay 
adjetivos ni expresiones de alta frecuencia (a excepción de gran diferencia) sino todo lo contrario, los adjetivos revelan una selección léxica elaborada.

\section{Resultados de las dimensiones morfológica, sintáctica y semántica}

En la Tabla 2, a continuación, se presentan los descriptivos para cuatro categorías de adjetivos que resultan particularmente interesantes desde el punto de vista del desarrollo: en la dimensión morfológica, los adjetivos derivados; en la dimensión sintáctica de función, los modificadores; en la dimensión sintáctica de posición, los prenominales y en una de las dimensiones semánticas, los adjetivos relacionales.

Tabla 2. Adjetivos derivados, modificadores, prenominales y relacionales. Medias grupales y DE (proporciones sobre el total de palabras del texto).

\begin{tabular}{|c|c|c|c|c|c|c|c|c|c|}
\hline & & \multicolumn{4}{|c|}{ GS medio-bajo } & \multicolumn{4}{|c|}{ GS Alto } \\
\hline & & Media & $\mathrm{DE}$ & Mín. & Máx. & Media & $\mathrm{DE}$ & Mín. & Máx. \\
\hline \multirow{3}{*}{ Derivado } & Séptimo & 0,0128 & 0,0090 & 0 & 0,0349 & 0,0081 & 0,0068 & 0 & 0,025 \\
\hline & Primero & 0,0116 & 0,0109 & 0 & 0,0357 & 0,0202 & 0,0098 & 0,0055 & 0,0435 \\
\hline & Tercero & 0,0160 & 0,0139 & 0 & 0,0526 & 0,0224 & 0,0121 & 0,0045 & 0,0413 \\
\hline \multirow{3}{*}{ Modificador } & Séptimo & 0,0251 & 0,0165 & 0 & 0,0698 & 0,0174 & 0,0088 & 0,0061 & 0,0321 \\
\hline & Primero & 0,0256 & 0,0169 & 0 & 0,0714 & 0,0350 & 0,0156 & 0,0075 & 0,0676 \\
\hline & Tercero & 0,0296 & 0,0176 & 0,0062 & 0,0680 & 0,0422 & 0,0183 & 0,0116 & 0,0916 \\
\hline \multirow{3}{*}{ Prenominal } & Séptimo & 0,0061 & 0,0065 & 0 & 0,0233 & 0,0033 & 0,0039 & 0 & 0,0123 \\
\hline & Primero & 0,0078 & 0,0089 & 0 & 0,0283 & 0,0104 & 0,0072 & 0 & 0,0242 \\
\hline & Tercero & 0,0048 & 0,0052 & 0 & 0,0188 & 0,0127 & 0,0089 & 0 & 0,0330 \\
\hline \multirow{3}{*}{ Relacional } & Séptimo & 0,0052 & 0,0058 & 0 & 0,0154 & 0,0021 & 0,0036 & 0 & 0,0106 \\
\hline & Primero & 0,0028 & 0,0058 & 0 & 0,0238 & 0,0086 & 0,0091 & 0 & 0,0273 \\
\hline & Tercero & 0,0064 & 0,0053 & 0 & 0,0194 & 0,0112 & 0,0097 & 0 & 0,0366 \\
\hline
\end{tabular}

\subsection{Adjetivos derivados}

Tal como se puede observar en la Tabla 2, el uso de adjetivos derivados morfológicamente tiende a aumentar con la edad/nivel escolar, aunque lo hace de maneras diferentes en los dos establecimientos. En el GS medio-bajo el incremento es leve (los menores usan $1,28 \%$ de adjetivos derivados sobre el total de palabras y los mayores usan 1,60\%) mientras que en el GS alto el uso de estos adjetivos en los estudiantes mayores $(2,24)$ triplica al de los menores $(0,81 \%)$. Consecuentemente, las pruebas ANOVA arrojan diferencias estadísticamente significativas para el factor edad/nivel escolar $(F(1,114)=6,884 ; p=, 0015)$ y los análisis 
posthoc revelan que las diferencias se dan esta vez exclusivamente entre los estudiantes de séptimo y los de tercero $(p=, 0010)$. El factor GS no arroja diferencias significativas $(p=, 0833)$ pero sí lo hace la interacción entre ambos factores $(F(1,114)=4,387 ; p=, 0146)$ lo que corrobora las diferentes trayectorias en el desarrollo de estos recursos en los dos establecimientos. Al igual que ocurría respecto del total de adjetivos, es la gran diferencia que se presenta entre los menores y los mayores del GS alto lo que determina la significatividad estadística por edad/nivel de toda la muestra, analizada de manera conjunta. Los dos primeros ejemplos expuestos más arriba, de séptimo básico, solo presentan un adjetivo derivado en total ("masiva"), mientras que el segundo texto presenta 9 derivados (incluyendo, como hemos dicho, los adjetivos propios y aquellos que son participios en función adjetival). Muchos de estos derivados son adjetivos deverbales, es decir, derivados de verbos (como "reconocido" o "importante") y otros son derivados de sustantivos o denominales (como "interesante" o "mundial"), que de manera generalizada son más escasos (Cutillas, Adjetivo y calidad 33). Estos resultados confirman la idea de que durante los años escolares aumenta el uso de palabras complejas morfológicamente, gracias al contacto con el lenguaje de la escuela. Los conocimientos morfológicos impactan, a su vez, en el desarrollo mismo del léxico, debido a las estrategias composicionales que permiten a los estudiantes inferir y recordar el significado de nuevas palabras (Anglin et al.150-152).

\subsection{Adjetivos con función modificadora}

Replicando la tendencia que se viene dando con los datos anteriores, los adjetivos modificadores aumentan ligeramente en el GS medio-bajo (de 2,51\% en séptimo a 2,96\% en tercero medio) y lo hacen notoriamente en el GS alto (de 1,7\% en séptimo a 4,2\% en tercero medio). Así, se repite el patrón de los adjetivos derivados: el factor edad/nivel escolar resulta nuevamente significativo $(F(1,114)=8,619 ; p=, 0003)$ a diferencia del factor GS $(p=, 1015)$, aunque sí lo es la interacción entre ambos $(F(1,114)=4,702 ; p=, 0109)$. Las pruebas posthoc para toda la muestra indican que las diferencias significativas por edad se encuentran entre los niveles del séptimo y tercero $(p=, 0002)$ y también entre séptimo y primero $(p=, 0319)$. Estos resultados confirman que, tal como se ha explicado en la introducción, los adjetivos con función modificadora son más frecuentes en los niños mayores que los atributos predicativos, debido a que aparecen más tardíamente en la ontogenia (Ninio 256) y además porque suelen relacionarse con construcciones sintácticas más complejas, particularmente cuando se trata de textos no narrativos (Biber y Gray 168). 
Artículo. Soledad Aravena, Riva Quiroga. “Desarrollo léxico tardío en textos explicativos de estudiantes secundarios: análisis de adjetivos”.

En los ejemplos anteriores también se ilustran estas diferencias: el segundo texto tiene solo un adjetivo con función de modificador, mientras que el tercero tiene 14 de 16. Cabe destacar, además, que los dos atributos predicativos de este texto están en oraciones con alta complejidad sintáctica. El primero de estos atributos es "múltiple"; está inserto en una cláusula subordinada concesiva y es modificado por una frase preposicional que a su vez contiene una cláusula adjetiva de relativo ("Si bien la utilización de esta red es múltiple en cuanto a la cantidad de recursos que tiene..."); el segundo es "partícipes", también elemento nuclear modificado por un complemento del adjetivo que a su vez contiene un adjetivo modificado por una frase preposicional y coordinado con otro adjetivo en una construcción elíptica ("para hacernos partícipes de comentarios interesantes en ciertas ocasiones y polémicos en otras"). Ello demuestra que, si bien los niños pequeños utilizan los atributos predicativos en construcciones simples, con la edad estos mismos recursos se manifiestan de manera muy sofisticada y confirma también el vínculo existente entre una mayor presencia de adjetivos con una mayor complejidad sintáctica (Ravid y Levie 30-45).

\subsection{Adjetivos en posición prenominal}

Los adjetivos prenominales, por su carácter no especificativo y, por tanto, más opcional e infrecuente que el de los demás adjetivos, constituyen un conjunto mucho menor en el total de adjetivos de todos los grupos, como puede verse en las cifras de la Tabla 2. Sin embargo, tienen un comportamiento muy particular: en el GS medio-bajo los estudiantes de séptimo básico utilizan 0,61 de estos adjetivos cada 100 palabras de texto, cifra que aumenta a $0,78 \%$ en primero medio y que luego, sorprendentemente, disminuye a 0,48\% en tercero medio. En el GS alto en tanto, los desempeños siguen una trayectoria evolutiva linealmente incremental, como en los apartados anteriores. Los menores utilizan 0,39 prenominales cada 100 palabras (un número bastante inferior que el de sus pares del mismo nivel en el otro establecimiento), cifra que aumenta a $0,72 \%$ en primero medio y luego a 0,89\% en tercero medio. En consecuencia, las pruebas de varianza para esta clase de adjetivos arrojan diferencias significativas para la edad/nivel escolar $(F(1,114)=4,773 ; p=, 0102)$, el grupo socioeconómico $(F(1,114)=3,949 ; p=$ ,0049) y la interacción entre estos factores $(F(1,114)=5,540 ; p=, 0050)$. Las comparaciones posthoc repiten la tendencia de resultados anteriores para el factor edad/nivel, es decir, las diferencias se presentan entre séptimo y tercero $(p=, 0172)$ y entre primero y tercero $(p=, 0317)$. Sin embargo, en el análisis de la interacción, estas pruebas revelan además una diferencia importante entre los terceros medios de ambos establecimientos $(p=, 0088)$. 
En un estudio anterior (en prensa) observamos que los adjetivos prenominales eran abundantes en las narraciones y particularmente en aquellas de los estudiantes mayores, quienes lograban dar más subjetividad y expresividad a sus historias mediante el uso de estos recursos (con ejemplos tales como "oscuros propósitos", "abominable objetivo" o "imparable fuerza"). Así, se pensó que los adjetivos prenominales tendrían una presencia menor en textos expositivos o informativos, debido a su carácter más subjetivo. Sin embargo, se constata que su presencia en este corpus de textos explicativos es similar y que su frecuencia también aumenta con la edad. Al respecto, es interesante constatar cómo los recursos lingüísticos se utilizan de manera diferente y crecientemente apropiada, según el género (Tolchinsky 235).

A continuación, se presenta un fragmento que ilustra bien este recurso en este tipo de texto. Como se puede apreciar, algunos adjetivos prenominales se usan con una cierta intención retórica que contribuye a dar un mayor énfasis, como ocurría en las narraciones. Es el caso de "gigantes estructuras" e "increíble juego" en el fragmento. Sin embargo, tal como se comentó antes para el Ejemplo 1, también hay otros usos de adjetivos prenominales que parecen menos opcionales o que, en cierta medida, constituyen expresiones de alta frecuencia dentro del género, como "constante cambio" y "diversos juegos" en este fragmento.

Ejemplo (4) tercero medio, GS alto (fragmento)

[...] Existe un modo multijugador el cual con el uso de internet reúne a distintos jugadores en servidores creados por estos mismos en los cuales dependiendo de qué es lo que se quiera hacer, se juntan jugadores para crear gigantes estructuras o competir entre estos. Por último este juego está en constante cambio ya que cada 3 o 4 meses es actualizado, lo cual rompe con la monotidad y rutina de los juegos. Por lo tanto este increíble juego toma factores de diversos juegos y los une para invitar a el jugador a crear, inventar o solo competir con otros el cual el único factor que limita a las personas es su imaginación y nos demuestra que un videojuego no necesita de buenas gráficas $\mathrm{y}$ violencia para poder divertirnos.

\subsection{Adjetivos relacionales}

En la dimensión más semántica de los adjetivos analizados, observamos que el uso de adjetivos relacionales tiende a aumentar con la edad/nivel escolar, aunque con distintas trayectorias en los dos establecimientos. Tal como se observa en la Tabla 2, en el GS medio-bajo su uso va de 0,52 adjetivos de este tipo cada 100 palabras en séptimo básico a 0,64\% en tercero medio, avance ligero que describe una trayectoria no lineal, puesto que en primero medio su uso desciende a 0,28\%. En el GS alto, los menores presentan 0,21\% (lo que equivale a decir que hay un adjetivo 
relacional cada 500 palabras, cifra dos veces inferior que la de sus pares del otro colegio), mientras que los mayores ascienden a un $1,12 \%$, lo que significa que su uso de los adjetivos relacionales quintuplica el de los alumnos de séptimo básico. De modo que las pruebas ANOVA confirman el efecto significativo del factor edad/nivel escolar para esta variable $(F(1,114)=$ $5,546 ; p=, 0050)$ y de la interacción entre la edad y el GS $(F(1,114)=4,867 ; p=, 0093)$. Este último factor, aunque revela diferencias importantes, no alcanza a mostrar significatividad estadística $(p=, 0569)$. Las comparaciones posthoc muestran que, tomando a los dos colegios juntos, las diferencias se presentan esta vez solo entre séptimo y primero $(p=, 0035)$. A pesar de su frecuencia relativamente escasa, los adjetivos relacionales son importantes en los textos de tipo expositivo/informativo como el del presente estudio, porque contribuyen a dar especificidad y precisión a los términos, lo que a su vez genera un discurso más objetivo. Se constata que su uso -más abundante en estos textos que en las narraciones de los mismos estudiantes analizadas en un estudio previo (autor, en prensa) - da cuenta del dominio del género que van alcanzando los jóvenes durante la escolaridad (Fang y Park 101). En efecto, los estudiantes menores de esta muestra, enfrentados a la escritura de este género en particular también los usan (a diferencia de lo que sucedía en sus narraciones) pero en menor medida y en expresiones de alta frecuencia, como la empleada en el Ejemplo (1) antes comentado ("comunicación masiva"). Los estudiantes mayores, en cambio, demuestran un mayor dominio de estos recursos característicos del género, tal como se puede apreciar en el fragmento que está a continuación, donde se usan para dar información específica e incluso especializada y revelan el dominio del hablante sobre el tema.

Ejemplo (5) tercero medio, GS alto (fragmento)

[...] La globalización cibernética ha permitido, a su vez, que no solo países en los cuales el idioma principal es inglés puedan compartir sus recetas, (aunque la mayoría de estas se encuentran en ese idioma) sino que también es posible encontrarse con contenido polaco, alemán, croata, entre otros. Gracias a esta web se ha desarrollado una creativa forma de compartir recetas que finalmente hacen a este sitio un lugar donde convergen diversas culturas. El ingreso, como se mencionó anteriormente, es muy fácil y apto para todas las edades, siendo muchos los beneficios que obtendrás si realmente estás interesado en el arte culinario.

En suma, todos estos resultados muestran que los distintos usos de adjetivos experimentan cambios importantes tanto en cuanto a la cantidad como en cuanto a las formas de uso, entre los 12 y los 17 años, es decir, entre el término de la enseñanza básica y durante casi toda la enseñanza media. Al respecto, es importante destacar, como se desprende de lo expuesto y se 
ilustra en el Gráfico 1, que los dos séptimos básicos (identificados con $\mathrm{S}$ en el gráfico) tienen un desempeño levemente diferente (sin significatividad estadística) en el uso de los distintos adjetivos, pero en beneficio de los estudiantes de GS medio-bajo. En efecto, este grupo muestra un desempeño superior al de sus pares del GS alto en todas las categorías analizadas, situación que se invierte drásticamente en los niveles superiores. Así, es posible afirmar que, al término de la enseñanza básica, los estudiantes de los dos GS no se distinguen mayormente, al menos en estos usos léxicos (y cuando lo hacen se verifica un mejor desempeño en el GS medio-bajo, que de hecho también escribe textos más largos). Comentaremos posibles explicaciones de este fenómeno en el siguiente apartado.

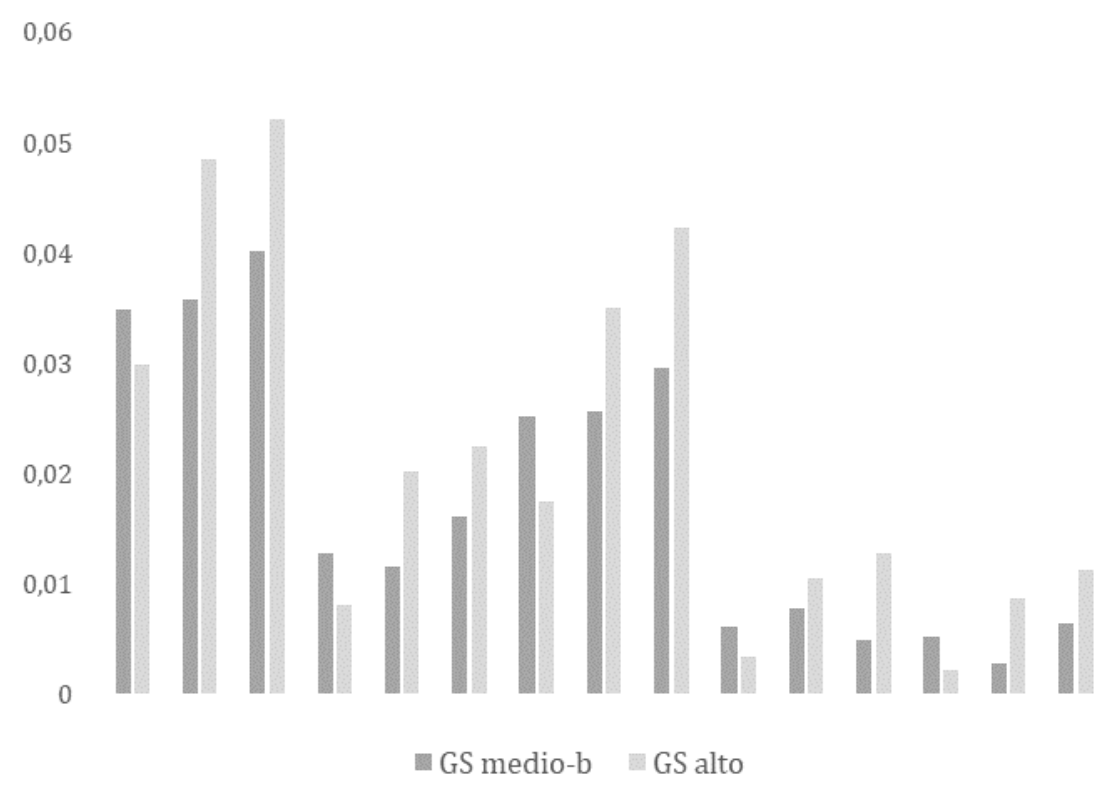

Gráfico 1. Todos los adjetivos por edad/nivel y GS; medias grupales.

\section{DISCUSIÓN}

En esta investigación se analizaron las frecuencias y las formas de uso de los adjetivos en textos explicativos escritos por estudiantes de entre 12 y 17 años y de dos grupos socioeconómicos. Al tratarse de una categoría léxica no estrictamente indispensable (a diferencia de sustantivos y verbos), los adjetivos se pueden considerar un buen indicador del desarrollo léxico y en particular de los desarrollos tardíos del léxico, es decir, aquellos que se presentan durante los años escolares.

La conclusión quizás más importante del estudio, debido a la consistencia del resultado del que se deriva, es que la edad/nivel escolar es un factor crítico en el número y el modo en que se utilizan los adjetivos, incluso cuando se trata de edades "avanzadas" (respecto del desarrollo 
del lenguaje) como las de este trabajo. En efecto, los resultados de las pruebas estadísticas muestran que todas las variables analizadas, es decir, la proporción de adjetivos totales, así como también las proporciones de adjetivos derivados, modificadores, prenominales y relacionales experimentan incrementos notables en estas edades. Algunos de estos cambios parecen producirse entre los 12 y los 14 años; es el caso de la proporción de adjetivos totales, la de adjetivos en función de modificadores y la de adjetivos en posición prenominal. Los cambios en las variables restantes, es decir, adjetivos derivados y adjetivos relacionales, se producen más tardíamente, después de los 14 años. Junto con las importantes diferencias en cuanto a las frecuencias de los adjetivos, también se observó que, a medida que los estudiantes avanzan en su escolaridad, utilizan adjetivos más complejos semánticamente y con una mayor amplitud de funciones (no tan solo calificar, sino que también categorizar, precisar y especializar una determinada entidad, entre otras), con creciente adecuación al género y en construcciones sintácticas cada vez más complejas. Este último punto no fue objeto del análisis cuantitativo del presente estudio, pero se revela como una proyección interesante para indagaciones futuras acerca del modo en que se relacionan la complejidad léxica y la sintáctica en ciertas etapas del desarrollo del lenguaje.

En segundo lugar, de los resultados de este estudio se concluye que el factor socioeconómico, por sí solo, tiene una incidencia menor que la edad o el nivel escolar en los desempeños léxicos de los estudiantes, al menos en lo que respecta a los usos adjetivales analizados. En efecto, el grupo socioeconómico solo resulta significativo en dos variables: la proporción de adjetivos totales de los textos y la de adjetivos prenominales, recursos significativamente más frecuentes en el GS alto, analizados los seis grupos de manera conjunta. El hecho de que una variable se vea afectada por el GS significa que no solo experimenta cambios por la mayor madurez cognitiva, linguística y vital de los hablantes (lo que correspondería al efecto de la edad) sino que también los experimenta producto de las prácticas letradas del hogar y de la escuela (Hoff 1368). Se confirma así que los adjetivos en general y los prenominales en particular son indicadores eficientes de un buen despliegue léxico, en consonancia con experiencias escolares y vitales enriquecidas linguiísticamente. Por otro lado, la interacción entre los factores edad y GS resulta significativa en todas las variables analizadas. Ello se traduce en que, aun cuando no haya diferencias entre los dos colegios para una determinada variable, por ejemplo, los adjetivos relacionales, de todas maneras, son significativamente diferentes sus trayectorias evolutivas. En muchos casos, se comprueba que el incremento entre séptimo y tercero medio es leve en el GS medio-bajo, mientras que en el GS 
alto el incremento entre estos niveles es notable. De hecho, es la gran diferencia entre el séptimo y el tercero medio del GS alto la que casi siempre determina la significatividad estadística por edad de toda la muestra. Y esto conduce a la siguiente conclusión, que requiere de un comentario un poco más extenso.

Tal como se ha adelantado más arriba, es interesante destacar el hecho de que en séptimo todos los desempeños del GS medio-bajo son mejores que los del GS alto ${ }^{1}$, aunque esto no llega a manifestarse en diferencias estadísticamente significativas. Se concluye entonces que, al término de la enseñanza básica, los estudiantes de ambos establecimientos se encuentran en condiciones similares desde el punto de vista de sus desarrollos léxicos, al menos de los recursos aquí comentados. Pero luego, al pasar a primero y después a tercero medio, se produce una diferencia drástica: mientras que los estudiantes del GS medio-bajo muestran incrementos muy leves o nulos en sus desempeños, los del GS alto experimentan cambios muy marcados en la cantidad y la calidad de sus usos de adjetivos. Pensamos que esto confirma la idea de que el comienzo de la adolescencia constituye otro hito importante en el desarrollo cognitivo y lingüístico (Tolchinsky 233-236) y que, por lo tanto, las experiencias de lenguaje oral y escrito que tengan los jóvenes en esta etapa van a determinar fuertemente sus logros al final de la escolaridad.

Asimismo, se puede plantear la hipótesis de que en estas edades "avanzadas" también se produce algo parecido al llamado "efecto Mateo" (Stanovich 36) fenómeno muy pertinente para comprender el ritmo que alcanzan ciertos aprendizajes, entre ellos, el de las palabras. A medida que los niños progresan en los años escolares, el incremento de la sofisticación del léxico les permite adquirir nuevas palabras más fácilmente y con menos necesidad de apoyo contextual. Como resultado, los niños que tienen estructuras léxicas más desarrolladas son más capaces de incrementarlas y, consecuentemente, enfrentan con mayores vocabularios una adquisición más rápida de nuevas palabras que servirán de base, a su vez, para nuevas expansiones de sus dominios conceptuales y semánticos. Además, el lenguaje escrito, cuyo dominio se consolida (teóricamente) al término de la enseñanza básica, se vuelve cada vez más central para ampliar el vocabulario, entre otras razones porque la habilidad para aprender palabras incidentalmente (o sea, durante la lectura) se incrementa con la edad (Cunningham y Stanovich 942; Nagy et al. 234237). Por ello, los niños y jóvenes que leen más ampliarán su léxico, lo que los habilitará para

1 Además de la interpretación posible que aquí se desarrolla, cabe señalar un dato no lingüístico que fue observado durante la recolección de los textos: los niños de séptimo del GS alto se comportaban -y se veían- de un modo más infantil que sus pares del GS medio-bajo, quienes parecían tener mucho más desplante y experiencia, lo que se vio reflejado en sus textos más largos y sus temas más juveniles. 
Artículo. Soledad Aravena, Riva Quiroga. "Desarrollo léxico tardío en textos explicativos de estudiantes secundarios: análisis de adjetivos".

continuar leyendo textos más largos y desafiantes. Esto también redunda en un nuevo beneficio, ya que como demuestran diversos estudios, la adquisición léxica se ve incrementada cuando el sistema cognitivo es exigido por la complejidad de las representaciones semánticas (Dockrell y Messer 50-51). En síntesis, los estudiantes que tengan la oportunidad de participar sistemáticamente de instancias comunicativas (conversaciones, exposiciones y, sobre todo, muchas lecturas narrativas y no narrativas) densas informativamente y sobre tópicos diversos y desafiantes, ampliarán sus vocabularios y alcanzarán mayores logros en lectura y rendimiento escolar (Verhoeven y Perfetti 2-3). Sin duda la familia y la escuela tienen un papel muy importante que cumplir en este "círculo virtuoso" que implica al aprendizaje de vocabulario y las prácticas comunicativas y lectoras en los niños y adolescentes.

El presente estudio deja plateadas nuevas inquietudes que sería interesante abordar en trabajos futuros; en particular, la relación que se establece entre la complejidad léxica y la sintáctica a lo largo del desarrollo y también, a la luz de los resultados actuales, la necesidad de buscar e implementar formas de intervención pedagógica orientadas al incremento del léxico. Cualquier intento en este sentido tendría que incluir, por cierto, la lectura y la escritura de diferentes tipos de textos, porque hemos vuelto a comprobar que el desarrollo lingüístico durante los años escolares es impulsado estrechamente por el dominio de los géneros. Sin embargo, se requiere además una enseñanza focalizada del léxico, hoy ausente o al menos feble en los currículos y las aulas escolares que refuerce los aprendizajes incidentales mediante la incorporación sistemática y controlada de nuevas palabras durante toda la escolaridad.

*Este estudio ha sido posible gracias al financiamiento del programa FONDECYT (Proyecto 11110191). 


\section{REFERENCIAS BIBLIOGRÁFICAS}

Ameel, Eef, Barbara Malt, and Gert Storms. "Object Naming and Later Lexical Development: From Baby Bottle to Beer Bottle." Journal of Memory and Language 58.2 (2008): 262-285. https://doi.org/10.1016/j.jml.2007.01.006

Anglin, Jeremy M., George A. Miller, and Pamela C. Wakefield. "Vocabulary Development: A Morphological Analysis." Monographs of The Society for Research in Child Development 58.10 (1993): i-186.

Berman, Ruth, Hrafnhildur Ragnarsdóttir, and Sven Strömqvist. "Discourse Stance: Written and Spoken Language." Written Language y Literacy 5.2 (2002): 255-289. https://doi.org/10.1075/wll.5.2.06ber

Biber, Douglas, and Bethany Gray. Grammatical Complexity in Academic English: Linguistic Change in Writing. Cambridge: Cambridge University Press, 2016.

Bloom, Paul. How Children Learn the Meanings of Words. Massachusetts: MIT press, 2000.

Bosque, Ignacio. "Sobre las diferencias entre los adjetivos relacionales y los calificativos." Revista Argentina de Lingüística 9 (1993): 9-48

Bosque, Ignacio, and Carme Picallo. "Postnominal Adjectives in Spanish DPs." Journal of Linguistics 32.2 (1996): 349-385. https://doi.org/10.1017/S0022226700015929

Clark, Eve V. First Language Acquisition. Cambridge: Cambridge University Press, 2009.

Cuadrado, Luis Alberto Hernando. "Gramática y estilística de la posición del adjetivo en español”. Didáctica. Lengua y Literatura 7.73 (1995): 73-87. https://core.ac.uk/download/pdf/38833811.pdf

Cunningham, Anne E., and Keith E. Stanovich. "Early Reading Acquisition and its Relation to Reading Experience and Ability 10 Years Later." Developmental Psychology 33.6 (1997): 934945.

Cutillas, Laia. "Un texto, un buen texto, un texto mejor: los adjetivos y la calidad de los textos." Textos de didáctica de la lengua y la literatura 65 (2014): 28-36.

https://dialnet.unirioja.es/servlet/articulo?codigo $=4678694$

- - -. L'ús dels adjectius al llarg del desenvolupament tardà del català. 2017. Tesis Doctoral no publicada, Universidad de Barcelona. http://hdl.handle.net/2445/118832

Cutillas, Laia, and Liliana Tolchinsky. "Use of Adjectives in Catalan: A Morphological Characterization in Different Genres and Modes of Production Through School-Age Development." First Language 37.1 (2017): 58-82. https://doi.org/10.1177/0142723716673957

Demonte, Violeta. "El adjetivo: clases y usos. La posición del adjetivo en el sintagma nominal." Gramática descriptiva de la lengua española Vol. 1, editado por Ignacio Bosque y Violeta Demonte, 1999, pp. 129-215. 
Artículo. Soledad Aravena, Riva Quiroga. "Desarrollo léxico tardío en textos explicativos de estudiantes secundarios: análisis de adjetivos".

Dockrell, Julie E., and David Messer. "Lexical Acquisition in the Early School Years." Language Development Across Childhood and Adolescence, edited by Ruth Berman, Amsterdam: John Hopkins, 2004. 35-52.

Faul, Franz, et al. "G* Power 3: A Flexible Statistical Power Analysis Program for the Social, Behavioral, and Biomedical Sciences." Behavior Research Methods 39.2 (2007): 175-191. https://doi.org/10.3758/BF03193146

Fang, Z., Park, J. “Adolescents' Use of Academic Language in Informational Writing”. Reading and Writing 33 (2020): 97-119. https://doi.org/10.1007/s11145-019-09937-8

Hansen, Pernille. "What Makes a Word Easy to Acquire? The Effects of Word Class, Frequency, Imageability and Phonological Neighbourhood Density on Lexical Development." First Language 37.2 (2017): 205-225. https://doi.org/10.1177/0142723716679956

Hoff, Erika. "The Specificity of Environmental Influence: Socioeconomic Status Affects Early Vocabulary Development Via Maternal Speech." Child Development 74.5 (2003): 1368-1378. https://doi.org/10.1111/1467-8624.00612

Lively, Scott E. et al. "Spoken Word Recognition: Research and Theory”. Handbook of psycholinguistics, editado por Morton Ann Gernsbacher, Academic Press: 1994. 265-301.

Llauradó, Anna, and Liliana Tolchinsky. "Growth of Text-Embedded Lexicon in Catalan: from Childhood to Adolescence." First Language 33.6 (2013): 628-653. https://doi.org/10.1177/0142723713508861

MacWhinney, Brian. The CHILDES Project Tools for Analyzing Talk-Electronic Edition. Lawrence Erlbaum Associates, 2014. http://citeseerx.ist.psu.edu/viewdoc/download?doi=10.1.1.687.5652yrep=rep1 ytype=pdf

McNally, Louise, and Christopher Kennedy, eds. Adjectives and Adverbs: Syntax, Semantics, and Discourse. Oxford: Oxford University Press, 2008.

Millán Chivite, Alberto. "Sintaxis del adjetivo español: orientación didáctica." Cauce 10 (1987): 201-231. http://hdl.handle.net/11441/32202

Nagy, William E., Patricia A. Herman, and Richard C. Anderson. "Learning Words from Context." Reading Research Quarterly 20.2 (1985): 233-253.

https://www.jstor.org/stable/747758

Nelson, Katherine. "Some Attributes of Adjectives Used by Young Children." Cognition 4.1 (1976): 13-30. https://doi.org/10.1016/0010-0277(76)90008-1

Neuendorf, K. The content analysis guidebook. London: Sage, 2002.

Ninio, Anat. "Young Children's Difficulty with Adjectives Modifying Nouns." Journal of Child Language 31.2 (2004): 255-285. https://doi.org/10.1017/S0305000904006191 
Nippold, Marilyn. Later Language Development: School-Age Children, Adolescents, and Young Adults (4 ${ }^{\text {th }}$ Ed.). Pro-Ed, 2016.

Real Academia Española (RAE). Nueva gramática de la lengua española. Madrid: Espasa Libros, 2010.

Ravid, Dorit. "Derivational Morphology Revisited." Language Development Across Childhood and Adolescence, editado por Ruth Berman, 2004. 53-83.

- - -. "Semantic Development in Textual Contexts During the School Years: Noun Scale Analyses." Journal of Child Language 33.4 (2006): 791-821.

https://doi.org/10.1017/S0305000906007586

Ravid, Dorit, and Ronit Levie. "Adjectives in the Development of Text Production: Lexical, Morphological and Syntactic Analyses." First Language 30.1 (2010): 27-55.

https://doi.org/10.1177/0142723709350529

Serrat, Elisabet, Mònica Sanz-Torrent, Iris Badia, Eva Aguilar, Raquel Olmo, Fernanda Lara, Llorenç Andreu y Miquel Serra. "The Relationship between Lexical Learning and Grammatical Development". Journal for the Study of Education and Development, 33:4 (2010): 435-448. https://doi.org/10.1174/021037010793139590

Stanovich, Keith E. "Matthew Effects in Reading: Some Consequences of Individual Differences in the Acquisition of Literacy." Journal of Education 189.1-2 (2009): 23-55.

https://doi.org/10.1177/0022057409189001-204

Stemler, Steven E. "A Comparison of Consensus, Consistency, and Measurement Approaches to Estimating Interrater Reliability." Practical Assessment, Research, and Evaluation 9.1 (2004): 4. https://scholarworks.umass.edu/cgi/viewcontent.cgi?article $=1137 \mathrm{ycontext}=$ pare

Tolchinsky, Liliana. "The Nature and Scope of Later Language Development." Language Development Across Childhood and Adolescence, edited by Ruth Berman. Amsterdam: John Benjamins, 2004: 233-247.

Tyler, Andrea, and William Nagy. "The acquisition of English Derivational morphology." Journal of memory and language 28.6 (1989): 649-667. https://doi.org/10.1016/0749-596X(89)90002-8

Verhoeven, Ludo, and Charles A. Perfetti. "Introduction to this Special Issue: Vocabulary Growth and Reading Skill." Scientific Studies of Reading 15.1 (2011): 1-7. https://doi.org/10.1080/10888438.2011.536124 